\section{Effect on mental health issues during the COVID-19 pandemic}

Catering to just under 29000 registered patients, I as a GP have been seeing a significant rise in patients seeking help with mental health-related issues. The COVID-19 pandemic has seen this increase in demand and I believe it is due to three main factors. The disruption in routine has affected the greater population, with the gravest ramifications for those patients who are mentally vulnerable. COVID-19 has seen many of these patients support systems crumble, with grave impacts on mental health, and therefore possible increase in suicide rates. Second, loss of income and a lack of money is a major stressor for most people; however, the vulnerable population is more likely to be furloughed and made redundant as the start of a downward spiral, resulting in further distress. Lastly, loneliness is a significant factor due to the stoppage of interaction with extended family and friends, and the reduction of face-toface contact with clinicians. Although this issue has been highlighted in the news, the full-blown effect of this really hit me when the total number of mental health-related consultations almost doubled during this pandemic. This was extremely frustrating to me, as GPs often see the patient's health as their holistic wellbeing, and the mental and emotional health of patients during the pandemic were not met. All three of these factors contribute to the decline in a patient's mental health, sometimes regrettably leading to self-harm and suicide. The coronavirus has taken many lives because of the physical decline in patients' health, but it may have or will also have claimed many lives because of the emotional, financial, and psychological stress it is continuously causing. We as GPs must strive to ensure that the long-term implications on patients' mental health due to COVID-19 are minimised as much as possible.

Parag Pandya,

GP Partner, Swanscombe \& Bean

Partnership, Kent.

Email: pandyalanhs.net

DOI: https://doi.org/ 10.3399/bjgp20X711857

\section{Emerging from COVID- 19: prioritising the burden of loneliness in older people}

Public health responses to COVID-19 have mandated the social isolation of millions of older people globally. While reducing viral transmission, this has undoubtedly exacerbated the pre-existing pandemic of loneliness among older adults, amplifying the associated burden of physical and mental ill-health. ${ }^{1}$ As governments begin to unlock their societies, they now have an opportunity to re-prioritise this longstanding public health emergency.

While those aged over 75 are at increased risk of severe COVID-19 disease and death, ${ }^{2,3}$ older adults are also vulnerable to the health impacts of prolonged isolation. Loneliness is often stigmatised, trivialised, or ignored, despite its strong association with physical and psychological morbidity, and premature mortality. ${ }^{4}$ Such marginalisation is particularly prevalent among older adults, representing an increasingly urgent public health issue in the ageing global population. ${ }^{1}$

As societies emerge from lockdown public health strategies must balance the risk of severe COVID-19 disease (for both older people and health services) against the burden of morbidity and mortality induced by prolonged isolation. Without a vaccine, viral transmission is expected to increase, posing greatest risk to older adults. This is reflected in governmental guidance, which continues to advise older people to stay at home and avoid contact with others outside their household. ${ }^{5}$ However, public health measures must avoid further marginalising of ageing communities. For example, relaxed measures permitting small groups from different households to congregate outdoors may exclude many older adults because of mobility limitations. Friends and family are still prevented from visiting older relatives at home, while daycare venues, community centres, and places of worship remain closed, denying many older adults their only sources of social contact.

There is urgent need for evidence-based discourse around minimising the risk of contagion in older adults while directly addressing the harms of loneliness The possibility of limited social contact, such as with friends, family, or voluntary organisations, mitigated by PPE and serological testing, should be considered along with further strengthening of telephone and digital communication links.

This moment represents an opportunity to shape the way loneliness in older adults is tackled, and trigger meaningful change in how this public health emergency is addressed beyond the pandemic.

Richard Armitage,

Division of Epidemiology \& Public Health, University of Nottingham, Nottingham City Hospital, Nottingham.

Email: msxra37@nottingham.ac.uk

Laura B Nellums,

Division of Epidemiology \& Public Health, University of Nottingham, Nottingham City Hospital, Nottingham.

Email: Laura.Nellumsanottingham.ac.uk

\section{REFERENCES}

1. Gerst-Emerson K, Jayawardhana J. Loneliness as a public health issue: the impact of loneliness on health care utilization among older adults. Am J Public Health 2015; 105(5): 1013-1019.

2. Public Health England. Disparities in the risk and outcomes of COVID-19. 2020. https://assets. publishing.service.gov.uk/government/uploads/ system/uploads/attachment_data/file/889861/ disparities_review.pdf (accessed 9 Jul 2020).

3. Office for National Statistics. Deaths involving COVID-19, England and Wales: deaths occurring in April 2020. 2020. https://www ons.gov.uk/peoplepopulationandcommunity/ birthsdeathsandmarriages/deaths/bulletins/ deathsinvolvingcovid19englandandwales/ deathsoccurringinapril2020 (accessed 9 Jul 2020).

4. Cacioppo JT, Cacioppo S. The growing problem of loneliness. Lancet 2018; 391(10119): 426. DOI https://doi.org/10.1016/S0140-6736(18)30142-9.

5. Cabinet Office. Staying alert and safe (social distancing). 31 May 2020. https://www.gov.uk/ government/publications/staying-alert-and-safesocial-distancing/staying-alert-and-safe-socialdistancing\#clinically-vulnerable-people [accessed 9 Jul 2020]

DOI: https://doi.org/10.3399/bjgp20X711869 